\title{
OUTPATHS OF ARCS IN REGULAR 3-PARTITE TOURNAMENTS
}

\author{
Qiaoping Guo and Wei Meng \\ School of Mathematical Sciences \\ Shanxi University, Taiyuan, 030006, China \\ e-mail: guoqp@sxu.edu.cn \\ mengwei@sxu.edu.cn
}

\begin{abstract}
Guo [Outpaths in semicomplete multipartite digraphs, Discrete Appl. Math. 95 (1999) 273-277] proposed the concept of the outpath in digraphs. An outpath of a vertex $x$ (an arc $x y$, respectively) in a digraph is a directed path starting at $x$ (an arc $x y$, respectively) such that $x$ does not dominate the end vertex of this directed path. A $k$-outpath is an outpath of length $k$. The outpath is a generalization of the directed cycle. A $c$-partite tournament is an orientation of a complete $c$-partite graph.

In this paper, we investigate outpaths of arcs in regular 3-partite tournaments. We prove that every arc of an $r$-regular 3-partite tournament has 2- (when $r \geq 1$ ), 3- (when $r \geq 2$ ), and 5-, 6-outpaths (when $r \geq 3$ ). We also give the structure of an $r$-regular 3-partite tournament $D$ with $r \geq 2$ that contains arcs which have no 4-outpaths. Based on these results, we conjecture that for all $k \in\{1,2, \ldots, r-1\}$, every arc of $r$-regular 3-partite tournaments with $r \geq 2$ has $(3 k-1)$ - and $3 k$-outpaths, and it has a $(3 k+1)$ outpath except an $r$-regular 3-partite tournament.
\end{abstract}

Keywords: multipartite tournament, regular 3-partite tournament, outpaths.

2010 Mathematics Subject Classification: 05C20, 05C38.

\section{REFERENCES}

[1] J. Bang-Jensen and G. Gutin, Digraphs: Theory, Algorithms and Applications, 2nd Edition (Springer, London, 2009). https://doi.org/10.1007/978-1-84800-998-1

[2] L. Cui and Q. Guo, Outpaths of arcs in almost regular multipartite tournaments, Acta Math. Appl. Sin. (Chinese Ser.) 39 (2016) 310-317. 
[3] Q. Guo and L. Cui, Outpaths of all length of an arc in regular multipartite tournaments, Appl. Math. J. Chinese Univ. (Chinese Ser.) 29 (2014) 288-294.

[4] Y. Guo, Outpaths in semicomplete multipartite digraphs, Discrete Appl. Math. 95 (1999) 273-277.

https://doi.org/10.1016/S0166-218X(99)00080-3

[5] L. Volkmann, Multipartite tournaments: a survey, Discrete Math. 307 (2007) 30973129. https://doi.org/10.1016/j.disc.2007.03.053

[6] G. Xu, S. Li, Q. Guo and H. Li, Notes on cycles through a vertex or an arc in regular 3-partite tournaments, Appl. Math. Lett. 25 (2012) 662-664. https://doi.org/10.1016/j.aml.2011.09.075

[7] G. Zhou and K. Zhang, Outpaths of arcs in multipartite tournaments, Acta Math. Appl. Sin. (Engl. Ser.) 17 (2001) 361-365.

Received 25 August 2018

Revised 9 March 2019

Accepted 9 March 2019 